\title{
LAS COMUNIDADES DE INSECTOS DEL PARQUE REGIONAL DE LA CUENCA ALTA DEL MANZANARES (CENTRO DE ESPAÑA): ESTADO DE CONSERVACIÓN
}

\author{
J. Martín Cano, J.M. Ferrín, E. García-Barros, A. García-Ocejo, \\ P. Gurrea, M.J. Luciáñez, M.L. Munguira, F. Pérez Barroeta, \\ M. Ruiz Ortega, M.J. Sanz Benito, J.C. Simón y J.L. Viejo (*)
}

\begin{abstract}
RESUMEN
El Parque Regional de la Cuenca Alta del Manzanares (PRCAM) es un área protegida del norte de Madrid. En el se han elegido ocho puntos representativos de sus ecosistemas y del uso del territorio. En estos puntos se han estudiado las comunidades de cuatro grupos de insectos: Colémbolos, Crisomélidos, Curculiónidos y Mariposas.

El objetivo del estudio es valorar el estado de conservación de los ecosistemas del parque, y el grado de paralelismo de la información suministrada por los cuatro grupos de insectos sobre unas mismas áreas.

Los resultados indican que los ecosistemas del Parque están muy alterados. El grado de deterioro es mayor en las zonas de menor altitud.

Por otro lado las comunidades de estos cuatro grupos de insectos responden de formas distintas ante situaciones idénticas. De ello se deduce la importancia de tener en cuenta varios grupos de organismos a la hora de manejar o valorar la diversidad biológica de un zona.

Palabras clave: diversidad de insectos, conservación, manejo de áreas protegidas, Parque Regional de la Cuenca Alta del Manzanares.
\end{abstract}

\section{ABSTRACT \\ The insect communities in the Parque Regional de la Cuenca Alta del Manzanares (Central Spain): conservation status.}

The Parque Regional de la Cuenca Alta del Manzanares (PRCAM) is a protected area at the North of Madrid. Eight sampling sites were selected representing different ecosystems and land uses. In these sites we studied the communities of four insect groups: Collembolla, Chrysomelida, Curculionoidea and Papilionoidea and Hesperioidea.

The objetive of the study was to assess the conservation status of the Park ecosystems and the paralelism of the results in these four insect groups.

The results show that the Park ecosystems are heavily disturbed. The lowland areas being damaged to a greater extent.

On the other hand the four groups have a different behaviour in similar conditions. This shows the importance of gathering information from different groups in order to assess or manage the biological diversity of an area.

Key words: insect diversity, conservation, management of protected areas, Parque Regional de la Cuenca del Manzanares.

* Departamento de Biología; Universidad Autónoma de Madrid; Campus de Cantoblanco, 28049 MADRID 


\section{Introducción}

La constitución de áreas protegidas obedece a múltiples motivos, entre los que destacan, además de los estrictamente conservacionistas, los aspectos sociales, económicos o culturales; muchas veces éstos son tan importantes o más que los primeros a la hora de fijar límites, usos o grado de protección. Normalmente la creación y declaración de zona protegida se apoya en algún aspecto sobresaliente, como puede ser la presencia de alguna o algunas especies de gran interés, un paisaje, un fenómeno geológico o un ecosistema singular. Con frecuencia los estudios previos, caso de existir, se refieren de forma casi exclusiva a ese aspecto central.

Al proponer la protección de una zona, los invertebrados en general, y los insectos en particular han sido ignorados en la mayoría de los casos. La simple enumeración de los parques nacionales españoles o europeos, o las declaraciones de las autoridades en materia de conservación pone de manifiesto este hecho. En los centros de recepción de visitantes, en los catálogos y publicaciones explicativas las referencias a los insectos son relativamente escasas. Paradójicamente, sin embargo, tanto por el número de especies, como por su grado de especialización en los ecosistemas, los insectos constituyen elementos indispensables para conocer el estado de una zona. En la actualidad se poseen amplios conocimientos de los requerimientos ecológicos de muchos grupos y especies de insectos y se ha puesto de manifiesto que son muy sensibles a los cambios del medio (Erhardt \& Thomas, 1991); por lo tanto resultan idóneos para valorar, desde el punto de vista ecológico y conservacionista, las áreas protegidas.

Pero este mismo elevado número de especies hace que un estudio que abarque al conjunto de los invertebrados sea una tarea difícil, que requiere muchos años de trabajos, dada la dificultad de convocar a todos los expertos necesarios para hacer simplemente un censo de las especies de todos los grupos (Margalef, 1991). Así, se han propuesto sistemas alternativos, que pretenden alcanzar un conocimiento satisfactorio de la biodiversidad, con un mínimo de recursos, o sea sin necesidad de acudir a los especialistas (Oliver \& Beattie, 1993).
Pero la gestión de un ecosistema requiere un amplio conocimiento del mismo, del conjunto de las especies que lo integran. Manejos que no están basados en un profundo conocimiento de la taxonomía de las especies de la zona pueden producir efectos contrarios a los deseados. Así, por beneficiar a especies relativamente comunes se pueden poner en peligro especies muy valiosas y amenazadas, simplemente por pertenecer a grupos menos populares o menos conocidos desde el punto de vista taxonómico (Daugherty et al., 1990).

En general no es suficiente para fijar una gestión adecuada de los hábitats naturales basarse exclusivamente en los vertebrados, pues hay factores que influyen de forma importante en las poblaciones de invertebrados, y que simultáneamente tienen poca importancia en las poblaciones de vertebrados, y viceversa (Murphy \& Wilcox, 1986). En el Reino Unido, las tasas de extinción debidas a la fragmentación del hábitat son mayores en invertebrados que en vertebrados (Thomas \& Morris, 1994).

El objetivo de este trabajo es dar a conocer los datos faunísticos y ecológicos de cuatro grupos de artrópodos diferentes en su biología y ecología. Estos cuatro grupos de insectos comprenden dos familias de Coleópteros fitófagos, los Crisomélidos y los Curculiónidos, siendo esta última familia la que cuenta con mayor número de especies del Reino Animal. Otro grupo, los Colémbolos, es uno los componentes más importantes y numerosos de la fauna edáfica, y finalmente, el último grupo, las Mariposas, representa el grupo de invertebrados más populares, y quizás del que se conozcan mejor los aspectos biológicos y ecológicos. Las diferencias y las similitudes que se dan en la estructura y composición de sus taxocenosis, las posibles correlaciones de los parámetros de los distintos taxones entre sí y con los del medio físico, se utilizarán como indicadores para valorar el estado de conservación del Parque Regional de la Cuenca Alta del Manzanares (PRCAM), área protegida del centro de la península Ibérica, declarada actualmente Reserva de la Biosfera, y que cuenta con diferentes tipos de ecosistemas. 


\section{Material y Métodos}

Área de estudio.- El PRCAM fue creado en 1985, y en la actualidad comprende unas 40.000 hectáreas, que abarcan desde las cercanías de la ciudad de Madrid, a unos $800 \mathrm{~m}$ de altitud, hasta las cumbres de la Sierra de Guadarrama, a $2.383 \mathrm{~m}$ de altitud. Comprende por tanto desde territorios situados en el piso mesomediterráneo, con encinas y quejigos, hasta los pastizales del piso oromediterráneo, pasando por los melojares, pinares y piornales. En algunos valles hay además zonas de fresneda, que junto con los pequeños restos de los bosques de ribera y las comunidades rupícolas, ampliamente representadas en el Parque, constituyen los principales paisajes de la zona.

El Parque comprende desde zonas urbanas e industriales hasta zonas denominadas de protección integral. La mayor parte del territorio se encuentra en zonas de intenso aprovechamiento agrícola y ganadero, donde predominan los usos tradicionales de la tierra (pastoreo, carboneo), que conviven con actividades del sector terciario (usos turísticos, recreativos y deportivos), muy favorecidas por la declaración de zona protegida.

Para este estudio se han elegido ocho puntos representativos de los diferentes ecosistemas presentes en el Parque, así como de su estado de conservación y manejo actual. En cinco de estos pun- tos se han estudiado los cuatro grupos de insectos aquí considerados, mientras que en los tres restantes se han estudiado exclusivamente Coleópteros Crisomélidos y Curculiónidos y Lepidópteros. Los puntos seleccionados están recogidos en la Tabla I, donde figuran además las cuadrículas en coordenadas UTM, la altitud, y la vegetación potencial y actual de cada uno, y el uso que tienen actualmente dentro del Parque.

Métodos de muestreo.- Los grupos estudiados en este trabajo son: los Colémbolos edáficos (Collembola), los Coleópteros Crisomélidos (Coleoptera: Chrysomelidae) y Curculiónidos (Coleoptera: Curculionoidea) que viven sobre el matorral de genisteas y las Mariposas (Lepidoptera: Papilionoidea y Hesperioidea).

La recogida de las muestras se ha realizado de modo que quedaran representados los tres pisos bioclimáticos del Parque (mesomediterráneo, supramediterráneo y oromediterráneo).

Los Colémbolos han sido extraídos por el método Berlesse-Tullgren, a partir de muestras de tierra de cada uno de los puntos estudiados. En cada localidad se tomó un volumen de $250 \mathrm{~cm}^{3}$ en cada una de las estaciones del año. De cada muestra se hicieron 3 réplicas. Una vez extraídos, los ejemplares se montan en preparaciones semipermanentes para su observación e identificación. Las listas

Tabla I.- Localidades muestreadas en el Parque Regional de la Cuenca Alta del Manzanares. Situación (coordenadas UTM) y principales características.

Table I.- Study site in the Parque Regional de la Cuenca Alta del Manzanares. Location (UTM grid coordinates) and main features.

\begin{tabular}{lccllll} 
Localidad & CUTM & Altitud & $\begin{array}{c}\text { Vegetación } \\
\text { actual }\end{array}$ & $\begin{array}{c}\text { Vegetación } \\
\text { potencial }\end{array}$ & Uso actual & Término municipal \\
\hline La Nava & 30TVL2316 & 1920 & Piornal & piornal & pastoreo & Manzanares el Real \\
Hueco de San Blas & 30TVL2817 & 1550 & Pinar repoblado & pinar & forestal & Manzanares el Real \\
Soto del Real & 30TVL3016 & 1250 & Melojar y pastos & melojar & pastoreo & Soto del Real \\
Colmenar Viejo & 30TVK2799 & 950 & Encinar y jaral & encinar & pastoreo & Colmenar Viejo \\
Cañada del Prado & 30TVL3011 & 940 & Melojar y pastos & melojar & pastoreo & Manzanares el Real \\
La Botica & 30TVL2900 & 930 & Encinar y pastos & encinar & pastoreo & Colmenar Viejo \\
El Boalo & 30TVL2304 & 920 & Fresneda y pastos & fresneda & pastoreo & El Boalo \\
Embalse de Santillana & 30TVL3010 & 900 & Fresneda y pastos & fresneda & pastoreo & Manzanares el Real
\end{tabular}


de especies, así como los aspectos metodológicos, sistemáticos, faunísticos y biogeográficos están recogidos en Luciáñez \& Simón (1991) y Luciáñez et al. (1992).

Los Coleópteros Curculiónidos y Crisomélidos se han recogido siguiendo los métodos clásicos de mangueo y vareo de la vegetación, recogida directa, recogida de frutos y otras partes de las plantas para la extracción de la fauna en el laboratorio. Las listas de las especies de estos grupos presentes en el Parque, así como los aspectos metodológicos y biogeográficos se encuentran en García-Ocejo, Gurrea \& Petitpierre (1992), Sanz Benito, GarcíaOcejo \& Gurrea (1992) y García-Ocejo \& Gurrea (1993).

Las Mariposas se han censado siguiendo transectos preestablecidos en cada una de las áreas elegidas (Harding, Asher \& Yates, 1995; Pollard \& Yates, 1993). Los ejemplares de difícil identificación se recogían para su examen detallado en el laboratorio. Más detalles sobre los aspectos metodológicos y los catálogos de las especies están en Viejo et al. (1992) y García-Barros et al. (1993).

El material recogido de todos los grupos se encuentra depositado en las colecciones del Departamento de Biología de la UAM.

La diversidad (H') se ha calculado según índice de información de Shannon-Weaver, tomando logaritmos con base 2 para obtener los resultados en bites (Margalef, 1975). El índice de riqueza (R) empleado ha sido el de Margalef (Magurran, 1988).

$$
\begin{gathered}
\mathrm{H}^{\prime}=-\Sigma\left(\mathrm{p}_{\mathrm{i}} \log _{2} \mathrm{p}_{\mathrm{i}}\right), \Sigma \mathrm{p}_{\mathrm{i}}=1 \\
\mathrm{R}=(\mathrm{S}-1) / \mathrm{LnN}
\end{gathered}
$$

$\mathrm{p}_{\mathrm{i}}$ : probabilidad de la especie $\mathrm{i}$

$\mathrm{S}$ : número de especies

$\mathrm{N}$ : número de ejemplares

Para el cálculo de la correlación de los parámetros ecológicos (abundancia, número de especies, riqueza y diversidad) de las comunidades de insectos entre sí, se ha empleado el coeficiente de correlación de rangos de Spearman. En el caso de la correlación entre la altitud y estos mismos parámetros se ha utilizado el coeficiente de correlación producto-momento de Pearson (Sokal \& Rohl, 1979).

Se han empleado estos cuatro parámetros que miden de alguna forma la diversidad de las comunidades porque cada uno de ellos ofrece información diferente de la estructura de los ecosistemas, dos de ellos son mas simples (abundancia y número de especies) y los otros dos (riqueza y diversidad) dan una información mas compleja, y en cierta medida complemetaria (Margalef, 1975; Wolda, 1983; Magurran, 1988).

Tabla II. - Número de ejemplares en las muestras de cada localidad.

Table II.- Number of individuals from the samples in each locality.

\begin{tabular}{lcccc} 
Localidad & Mariposas & Curculiónidos & Crisomélidos & Colémbolos \\
\hline La Nava & 52 & 660 & 49 & 1910 \\
Hueco de San Blas & 194 & 714 & 84 & 3434 \\
Soto del Real & 286 & 2171 & 216 & 811 \\
Colmenar Viejo & 101 & 3 & 3 & 439 \\
Cañada del Prado & 453 & 144 & 12 & 396 \\
La Botica & 230 & 8 & 12 & \\
El Boalo & 112 & 25 & 118 & 6990 \\
Embalse de Santillana & 328 & 3727 & 505 &
\end{tabular}




\section{Resultados}

Considerando el número de individuos (Tabla II) los valores más elevados se dan en los melojares y en el pinar. En el melojar de Soto del Real para Crisomélidos y Curculiónidos, en el melojar de la Cañada del Prado para Mariposas, y en el pinar en el caso de los Colémbolos. Los valores menores se dan en los encinares y fresnedas y en el piornal. El encinar de Colmenar Viejo para Crisomélidos, la fresneda de El Boalo para Curculiónidos y Colémbolos, y el piornal para las Mariposas registran los valores mínimos. En conjunto la mayor abundancia de individuos se da en las localidades de los pisos altitudinalmente superiores, piornal, pinar y melojares, mientras que las menores abundancias se dan en los pisos de menor altitud, encinares y fresnedas. Sin embargo esto no es un comportamiento generalizado y paralelo en los grupos considerados. En la Tabla VIa se puede ver que las abundancias de los grupos estudiados no están correlacionadas.

Respecto al número de especies (Tabla III), los puntos con mayor número son igualmente el melojar de Soto del Real en Crisomélidos y Curculiónidos, y el pinar en Mariposas y Colémbolos. Los valores mínimos se encuentran también en el encinar de Colmenar Viejo para Curculiónidos y Crisomélidos, en esta misma localidad y en la fresneda de El Boalo para los
Colémbolos, y en el piornal para las Mariposas. Al igual que en el caso del número de individuos, las localidades de los pisos del piornal, pinar y melojar, con alguna excepción, tienen el mayor número de especies. Los encinares y las fresnedas son los más pobres. Pero el comportamiento de los cuatro grupos es relativamente diferente, solamente son significativos los coeficientes de correlación entre los Crisomélidos y los Curculiónidos, y entre estos últimos y los Colémbolos, Tabla VIb.

Los valores máximos de riqueza se dan en el pinar para Mariposas, Curculiónidos y Colémbolos, y en el melojar de Soto del Real para Crisomélidos. Los valores mínimos son para el encinar de Colmenar Viejo en los cuatro grupos (Tabla IV). Igualmente, en conjunto, los valores más altos se corresponden con las localidades de mayor altitud, piornales, pinares y melojares, y los más bajos con los encinares y fresnedas, excluyendo la fresneda del Embalse de Santillana, que tiene unos valores del índice de riqueza relativamente elevados. No hay correlaciones significativas entre los índices de riqueza de los grupos estudiados, Tabla VIc.

En el caso del índice de diversidad (Tabla V), los valores máximos se dan en el melojar de Soto del Real en Mariposas, Curculiónidos y Crisomélidos, y en el pinar en los Colémbolos. Los valores mínimos se han obtenido en el encinar de Colmenar Viejo para Mariposas, Curculiónidos y Crisomélidos y en la fresneda de El Boalo para

Tabla III.- Número de especies encontradas en cada localidad.

Table III. - Number of species from the samples in each locality.

\begin{tabular}{lcccc} 
Localidad & Mariposas & Curculiónidos & Crisomélidos & Colémbolos \\
\hline La Nava & 8 & 20 & 9 & 6 \\
Hueco de San Blas & 24 & 36 & 31 & 1 \\
Soto del Real & 19 & 40 & 4 & 17 \\
Colmenar Viejo & 10 & 10 & 6 & 24 \\
Cañada del Prado & 23 & 3 & 9 & 17 \\
La Botica & 17 & 2 & 9 & 47 \\
El Boalo & 14 & 11 & 47
\end{tabular}


Tabla IV.- Valores del índice de riqueza de Margalef (R).

Table IV.- Values for Margalef's richness index (R).

\begin{tabular}{lcccc} 
Localidad & Mariposas & Curculiónidos & Crisomélidos & Colémbolos \\
\hline La Nava & 1,77 & 2,93 & 2,05 & 4,63 \\
Hueco de San Blas & 4,36 & 5,33 & 1,13 & 5,15 \\
Soto del Real & 3,18 & 5,07 & 5,58 & 2,38 \\
Colmenar Viejo & 1,95 & 0,00 & 0,00 & 3,78 \\
Cañada del Prado & 3,59 & 1,81 & 1,21 & 2,67 \\
La Botica & 2,94 & 0,96 & 2,05 & \\
El Boalo & 2,75 & 1,44 & 0,84 & 8,36 \\
Embalse de Santillana & 3,62 & 3,10 & 3,33 &
\end{tabular}

los Colémbolos. En conjunto los melojares, el pinar y el piornal tienen valores más altos del índice de diversidad, pero la correlación sigue siendo baja, Tabla VId. Hay correlaciones significativas entre los índices de los grupos de fitofagos (Crisomélidos, Curculiónidos y Mariposas), mientras que los Colémbolos tienen unas variaciones en los valores de la diversidad no correlacionadas con las de los otros grupos.

Para el conjunto de los factores analizados (abundancia, $\mathrm{n}^{\mathrm{o}}$ de especies, índice de riqueza e índice de diversidad) se pueden separar los puntos de muestreo en dos conjuntos. El primero encabe- zado por el melojar de Soto del Real, seguido por el pinar del Hueco de San Blas, el melojar del Embalse de Santillana y finalmente el piornal de La Nava, donde la mayoría de parámetros tienen valores por encima de la media. Por otro lado están los puntos que tienen la mayoría de los factores con valores inferiores a la media, el primero de los cuales es el encinar de Colmenar Viejo, seguido por la fresneda de El Boalo, el melojar de la Cañada del Prado y el encinar de la finca La Botica.

Tabla V.- Valores del índice de diversidad de Shannon-Weaver (H').

Table V.- Values for Shannon-Weaver's diversity index (H').

\begin{tabular}{lcccc} 
Localidad & Mariposas & Curculiónidos & Crisomélidos & Colémbolos \\
\hline La Nava & 2,79 & 2,03 & 1,61 & 3,94 \\
Hueco de San Blas & 3,98 & 2,81 & 1,97 & 3,97 \\
Soto del Real & 4,03 & 3,07 & 3,33 & 3,19 \\
Colmenar Viejo & 2,01 & 0,00 & 0,00 & 3,41 \\
Cañada del Prado & 3,47 & 0,87 & 1,62 & 2,55 \\
La Botica & 2,89 & 1,06 & 2,35 & \\
El Boalo & 3,42 & 1,00 & 0,62 & 4,75 \\
Embalse de Santillana & 3,49 & 2,91 & 3,03 & 3,57
\end{tabular}


Tabla VI.- Correlación entre las abundancias, número de especies, riqueza y diversidad de los cuatro grupos de insectos estudiados. Coeficientes de correlación de Spearman r, entre paréntesis figura el número de casos.

ns: no significativo; *: $\mathrm{p}<0,05 ; * *: \mathrm{p}<0,01$.

Table VI.- Correlation between abudance, number of species, richness amd diversity in the four studied insect groups. Spearman's correlation coefficients $r$, in brackets the number of cases. ns: not significant; *: $\mathrm{p}<0,05$.

\begin{tabular}{cccc} 
a) INDIVIDUOS & Curculiónidos & Crisomélidos & Colémbolos \\
\hline $\begin{array}{l}\text { Mariposas } \\
\text { Curculiónidos }\end{array}$ & $0,238(8) \mathrm{ns}$ & $-0,060(8) \mathrm{ns}$ & $-0,200(5) \mathrm{ns}$ \\
Crisomélidos & & $0,455(8) \mathrm{ns}$ & $0,900(5) \mathrm{ns}$ \\
b) & & & $-0,100(5) \mathrm{ns}$ \\
ESPECIES & Curculiónidos & Crisomélidos & Colémbolos \\
\hline Mariposas & $0,429(8) \mathrm{ns}$ & $0,060(8) \mathrm{ns}$ & $0,359(5) \mathrm{ns}$ \\
Curculiónidos & & $0,807(8) *$ & $0,975(5) *$ \\
Crisomélidos & & & $0,718(5) \mathrm{ns}$ \\
c) RIQUEZA & Curculiónidos & Crisomélidos & Colémbolos \\
\hline Mariposas & $0,643(8) \mathrm{ns}$ & $0,252(8) \mathrm{ns}$ & $0,000(5) \mathrm{ns}$ \\
Curculiónidos & & $0,515(8) \mathrm{ns}$ & $0,900(5) \mathrm{ns}$ \\
Crisomélidos & & & $0,900(5) \mathrm{ns}$ \\
d) DIVERSIDAD & Curculiónidos & Crisomélidos & Colémbolos \\
\hline Mariposas & $0,738(8) *$ & $0,762(8) *$ & $0,500(5) \mathrm{ns}$ \\
Curculiónidos & & $0,833(8) *$ & $0,700(5) \mathrm{ns}$
\end{tabular}

\section{Discusión}

De los datos expuestos, hay dos puntos que llaman la atención. El primero es la tendencia que tienen los parámetros ecológicos estudiados a aumentar con la altitud, en algún caso con valores significativos estadísticamente (abundancia en Curculiónidos) o con valores muy próximos a la significación (en Colémbolos para la abundancia $\mathrm{p}=0,079$; para el número de especies $\mathrm{p}=0,068$; para la riqueza $\mathrm{p}=0,095$ y para la diversidad $\mathrm{p}=$ 0,093). El otro aspecto llamativo es la escasa o nula correlación que hay entre los valores de los parámetros ecológicos en los diferentes grupos taxonómicos estudiados.

En el primer punto, la variación de los parámetros ecológicos con la altitud, los datos obtenidos se pueden englobar en una tendencia generalizada de forma que las comunidades más ricas en especies e individuos, y con una mayor complejidad (mayores índices de diversidad y riqueza) se dan en los ecosistemas de mayor altitud (piornales, pinares y melojares), y las comunidades más pobres en especies e individuos en los lugares de menor altitud (encinares y fresnedas). Las Mariposas son el único grupo de los considerados cuya tendencia es a disminuir el número de individuos con la altitud, variación general en la biosfera, mientras que en Curculiónidos, Crisomélidos y Colémbolos tiende a aumentar el número de sus individuos, aunque de forma no significativa en los dos últimos grupos. Lo mismo sucede con el número de especies. Excepto en Mariposas, hay más especies conforme aumenta la altitud.

Estamos por lo tanto ante una situación inversa a la esperada. La norma general es que en un área, según aumenta la altitud disminuye el número de especies y la diversidad (Brown, 1988). En concreto en la Sierra del Guadarrama hay una pérdida de 
Tabla VII.- Correlación de los cuatro parámetros ecológicos estudiados (abundancia, número de especies, riqueza y diversidad) con la altitud, para cada grupo estudiado. Coeficientes de correlación de Pearson r, entre paréntesis figura el número de casos. ns: no significativo; $*: \mathrm{p}<0,05$.

Table VII.- Correlation with altitude of the four studied ecological parameters (abudance, number of species, richness and diversity) for each studied insect group. Pearson's correlation coefficients $r$, in brackets the number of cases. ns: not significant; *: $\mathrm{p}<0,05$.

\begin{tabular}{lcccc} 
ALTITUD & Mariposas & Curculiónidos & Crisomélidos & Colémbolos \\
\hline log individuos & $-0,553(8) \mathrm{ns}$ & $0,706(8) *$ & $0,458(8) \mathrm{ns}$ & $0,834(5) \mathrm{ns}$ \\
especies & $-0,274(8) \mathrm{ns}$ & $0,619(8) \mathrm{ns}$ & $0,212(8) \mathrm{ns}$ & $0,847(5) \mathrm{ns}$ \\
riqueza & $-0,167(8) \mathrm{ns}$ & $0,551(8) \mathrm{ns}$ & $0,115(8) \mathrm{ns}$ & $0,806(5) \mathrm{ns}$ \\
diversidad & $0,110(8) \mathrm{ns}$ & $0,443(8) \mathrm{ns}$ & $0,105(8) \mathrm{ns}$ & $0,813(5) \mathrm{ns}$
\end{tabular}

especies vegetales de 0,84 especies $/ 0,04 \mathrm{~m}^{2}$ por cada incremento de $100 \mathrm{~m}$ de altitud (Montalvo et al. 1993), que equivale a tres especies de plantas cada $100 \mathrm{~m}$ o 0,1 bit de diversidad cada $100 \mathrm{~m}$ (Montalvo et al., 1991). Igual sucede en Pirineos, donde conforme aumenta la altitud disminuye la diversidad de plantas, pájaros y ortópteros (Antor, Isern-Vallverdú \& Pedrocchi-Renault, 1991).

Las causas de la inversión de esta regla general en estos grupos de insectos pueden tener dos orígenes, natural y antrópico. Entre las causas de origen natural podemos señalar el déficit hídrico en las zonas bajas del Parque, y la pobreza y fragilidad de sus suelos. Como se desprende de la Tabla I, en el Parque en las altitudes comprendidas entre los 800 y $1.000 \mathrm{~m}$ aproximadamente, se alternan las zonas de encinas, con las de fresnos y melojos, con frecuentes inversiones altitudinales. Estas inversiones están relacionadas principalmente con la meteorología y las pequeñas variaciones en las características edáficas. En invierno no son raras las situaciones de inversión térmica, y en general, los suelos del Parque tienen una escasa capacidad de retención de agua y son muy frágiles ante los procesos erosivos. El déficit hídrico en la Sierra de Guadarrama a 1900 m tiene una duración media de tres meses (de julio a septiembre), pero a altitudes más bajas, como a $700 \mathrm{~m}$ alcanza los cinco meses (de junio a octubre) Montalvo et al. (1993). Pero podemos considerar que estas razones no deben ser las más importantes, ya que serían aplicables también a los vegetales, lo que no parece ser el caso. En concreto uno de los ecosistemas que han resultado más pobres es una de las fresnedas, las cuales se sitúan sobre suelos con un nivel freático alto, con lo cual el posible estrés hídrico queda amortiguado.

Otras causas serían las debidas a la actuación del hombre. Fundamentalmente la deforestación y adehesamiento casi generalizado de la mayor parte del territorio, y el sobrepastoreo. Estas prácticas han conducido a la desaparición del sotobosque y a la alteración del suelo, responsables del empobrecimiento de la fauna de Curculiónidos, Crisomélidos y Colémbolos singularmente en las zonas de menor elevación.

Este bajo número de especies y de individuos de Colémbolos en las zonas más bajas del Parque, y sobre todo su correlación positiva con la altitud indican una importante erosión y alteración del suelo en las zonas más bajas.

En el otro aspecto a destacar, la disparidad en los parámetros ecológicos en los grupos estudiados, se entiende como señala Margalef (1975), que la diversidad calculada sobre un grupo taxonómico cuyo espectro ecológico general sea amplio por tener miembros especializados en direcciones muy diversas, puede ser un buen indicador de la diversidad total. En este caso por lo tanto sería esperable que la diversidad de los cuatro grupos variase de forma correlativa, y fuese alta en las zonas del Parque más ricas o mejor conservadas, y tuviese valores menores, en los cuatro grupos, en las zonas del parque más pobres o en peor estado de conservación. Sin embargo, sólo las taxocenosis de Curculiónidos y Crisomélidos muestran un cierto paralelismo en sus parámetros. 
Una de las causas de esta disparidad puede deberse a los diferentes requerimientos ecológicos de los grupos estudiados. En este sentido, una de las condiciones que señala Margalef $(1975,1991)$ es que la diversidad esté calculada sobre grupos taxonómicos de amplio espectro ecológico por tener miembros especializados en direcciones muy diversas, porque la diversidad de un grupo cuyos componentes tengan unas posibilidades de vida más restringidas puede no estar correlacionada con la diversidad de grupos menos exclusivos o con la del conjunto. Y más concretamente, Margalef (op. cit.) señala que la diversidad de las poblaciones de Coleópteros está vinculada a etapas diferentes de la sucesión. La diversidad es menor en terrenos cultivados, y en vegetación abierta o pinares, y mayor en bosques de robles y hayas. Estas conclusiones, señala este mismo autor, son probablemente generalizables a Arácnidos, Colémbolos y otros grupos de Artrópodos, excepto grupos de condiciones del entorno específicos, como especies de Curculiónidos, Crisomélidos, etc., que están vinculadas a etapas diferentes de la sucesión.

El hecho de que los resultados faunísticos sean tan dispares en los diferentes grupos resalta lo importante que es no centrar la conservación de un espacio natural en un taxón concreto, ya que lo que puede resultar beneficioso para ese grupo puede no serlo para los demás. Los animales tenidos en cuenta para la declaración de espacios protegidos son casi siempre vertebrados (Murphy \& Wilcox, 1986), y tanto nuestros resultados sobre la pobreza del parque en estos grupos de insectos, como la disparidad de resultados en las distintas áreas y grupos, indican que es necesario tener en cuenta muchos más factores a la hora de decidir los manejos de un área concreta, así como la declaración de nuevos espacios protegidos.

A la escasa idoneidad de los vertebrados para tomar decisiones que afecten al conjunto de la fauna (Murphy \& Wilcox, 1986; Thomas \& Morris, 1994) hay que añadir, que según nuestros resultados, no hay evidencia de que los invertebrados, y en particular los insectos se comporten a nivel de comunidad como un conjunto homogéneo: diferentes taxones pueden verse influidos de formas muy distintas por una misma situación.
Como conclusión final podemos señalar que el Parque presenta una fauna entomológica empobrecida. Esto se pone de manifiesto por el menor número de especies con respecto a zonas adyacentes de características similares, incluida la misma Sierra de Guadarrama, en Mariposas (GarcíaBarros et al., 1993), Crisomélidos (García-Ocejo et al., 1992), Curculiónidos (Velazquez de Castro; Alonso Zarazaga \& Outerelo, 1990; Sanz Benito \& Gurrea, 1995) y Colémbolos (Luciáñez \& Simón, 1991 y Luciáñez et al., 1992). La falta o escasez de algunas especies emblemáticas como Parnasius apollo (L.) (Lepidoptera: Papilionidae), que parece haber sido abundante en diversas zonas del Parque en fechas relativamente recientes (Gómez-Bustillo \& Fernández-Rubio, 1974; Angel Simón Sorli, com. pers., 1993) refuerza esta hipótesis.

Finalmente, en las zonas bajas del Parque, por debajo de los $1.000 \mathrm{~m}$ aproximadamente, es necesaria una rigurosa política de restauración que permita la recuperación de su flora y fauna, ahora muy deterioradas. Las zonas de mayor altitud parecen mantener una diversidad más elevada, y posiblemente controlar la presión ganadera sea suficiente. La reciente introducción de la cabra montés en la zona es un factor a tener en cuenta, ya que puede alterar aún más el precario equilibrio en que se encuentran las zonas de mayor altitud del Parque.

\section{AGRADECIMIENTOS}

Este trabajo ha sido financiado por la DGICYT, proyectos PB 87/0095 y PB 90/0197. Agradecemos a los responsables del Parque Regional de la Cuenca Alta del Manzanares los permisos para realizar este estudio.

\section{Referencias}

Antor, R.J., Isern-Vallverdú, J. \& PedrocchiRenault, C., 1991. Analysis of community diversity in Pyrenean ecosystems (NE Spain) and their relationship with the altitudinal gradient and the environment organization. En: F. D. Pineda et al. (eds.) Diversidad Biológica/Biological Diversity. F. ArecesADENA/WWF-SCOPE, Madrid: 127-130. 
Brown, J.H., 1988. Species diversity. En: A.A. Myers \& P.S. Giller (eds.) Analytical Biogeography. Chapman and Hall, London: 57-89.

Castroviejo, S. et al. (Eds.) 1986 \& 1990. Flora ibérica. Plantas vasculares de la Península Ibérica e Islas Baleares. 1 \& 2. Real Jardín Botánico, C.S.I.C., Madrid. 575 \& 897 pp.

Daugherty, C.H., Cree, A., Hay, J.M. \& Thompson, A.M., 1990. Neglected taxonomy and continuing extinctions of tuatara (Sphenodon). Nature, 347: 177-179.

ERHARDT, A. \& THOMAS, J.A., 1991. Lepidoptera as indicators of change in the Seminatural Grasslands of Lowland up Upland Europe. En: N.M. Collins \& J.A. Thomas (Eds.) The Conservation of insects and their habitats. Academic Press, London: 213-236.

García-Barros, E., Martín, J., Munguira, M.L. \& VIEJO, J.L., 1993. Fauna de mariposas y macroheteróceros del Parque Regional de la Cuenca Alta del Manzanares, Madrid, España (Insecta: Lepidoptera). Shilap Rev. Lepid., 21(82): 119-129.

García-Ocejo, A. \& Gurrea, P., 1993. Endemismos ibéricos de Coleópteros Crisomélidos (Chrysomelidae) del Sistema Central. Parques regionales de la Sierra de Gredos y de la Cuenca Alta del Manzanares (España). Bol. R. Soc.esp. Hist. Nat. (Sec. Biol.), 90(1-4): 33-41.

García-Ocejo, A.; Gurrea, P. \& PetitPierre, E., 1992. Chrysomelidae (Coleoptera) de la Sierra de Gredos (Sistema Central) datos faunísticos, ecológicos y fenológicos. Misc. Zool., 16: 8192.

García-Ocejo, A.; Sanz Benito, M.J. \& Gurrea, P., 1992. Entomofauna del piso oromediterráneo del Parque Regional de la Cuenca Alta del Manzanares: Ecología de Coleópteros fitófagos de piornales. Bol. Soc. Port. Entomol., suplemento 3(2): 209-218.

Gómez-Bustillo, M. \& Fernández-Rubio, F., 1974. Mariposas de la Península Ibérica. I. ICONA, Madrid. 198 pp.

Harding, P.T., Asher, J. \& Yates, T.J., 1995. Butterfly monitoring 1 - recording the changes. En: A.S. Pullin (Ed.) Ecology and conservation of Butterflies. Chapman and Hall, London: 3-22.
LuCiáñez, M.J.; Martín, J.; Pérez, M. \& Simón, J.C., 1992. Contribución al estudio ecológico de la colembofauna en un robledal en la Comunidad de Madrid. En: A. Alemany (Ed.) Historia Natural'91. Universitat de les Illes Balears, Palma. Vol II: 203-210.

LuCiáÑEZ, M.J. \& Simón, J.C., 1991. Estudio de la variación estacional de la colembofauna en suelos de alta montaña en la Sierra de Guadarrama (Madrid). Misc. Zool., 15: 103-113.

MagurRan. A.E., 1988. Diversidad ecológica y su medición. Vedrá, Barcelona. 200 pp.

MARGAlef, R., 1975. Ecología. Ediciones Omega. Barcelona. $951 \mathrm{pp}$.

MARGALEF, R., 1991. Reflexiones sobre la diversidad y significado de su expresión cuantitativa. En: F.D. Pineda et al. (Eds.) Diversidad Biológica/Biological Diversity. F. ArecesADENA/WWF-SCOPE, Madrid: 105-112.

Montalyo, J., Casado, M.A., Levassor, C. \& PINEDA, F.D. 1991., Patrones de diversidad específica y fenotípica. En: F.D. Pineda et al. (Eds.) Diversidad Biológica/Biological Diversity. F. Areces-ADENA/WWF-SCOPE, Madrid: $117-120$

Montalvo, J., Casado, M.A., Levassor, C. \& PINEDA, F.D., 1993. Species diversity patterns in Mediterranean grasslands. J. Veg. Sci., 4: 213222.

MuRPHY, D.D. \& Wilcox, B.A., 1986. Butterfly Diversity in Natural Habitat Fragments: A test of the Validity of Vertebrate-Based Management. En: J. Verner, M.L. Morrison \& C.J. Ralph (Eds.) Wildlife 2000: Modeling Habitat Relationships of Terrestrial Vertebrates. The University of Wisconsin Press, Madison: 287-292.

Oliver, I. \& Beattie, A.J., 1993. A possible Method for the Rapid Assesment of Biodiversity. Conserv. Biol., 7, 3: 562-568.

Pollard, E. \& YATES, T.J., 1993. Monitoring Butterflies for Ecology and Conservation. Chapman and Hall, London. 274 pp.

Rivas-Martínez, S., 1987. Mapa de series de vegetación de España 1:400.000 y memoria. ICONA, Madrid. 268 pp. 
Sanz Benito, M.J., García-Ocejo, A. \& GurRea, P., 1992. Coleópteros fitófagos (Curculionoide y Chrysomelidae) del endemismo ibérico Genista cinerascens Lange en el Parque Regional de la Cuenca Alta del Manzanares (Sistema Central). Bol. Soc. Port. Entomol., suplemento 3(1): 363-372.

Sanz Benito, M.J. \& Gurrea, P., 1995. Curculionoidea (Coleoptera) de la Sierra del Guadarrama. Avances en Entomología Ibérica, Museo Nacional de Ciencias Naturales y Universidad Autónoma de Madrid, Madrid: 279-300.

SoKal, R.R. \& RoHL, F.J., 1979. Biometría. H. Blume Ediciones, Madrid. 832 pp.

Thomas, J.A. \& Morris, M.G., 1994. Patterns, mechanisms and rates of extinction among invertebrates in the United Kingdom. Philos. Trans. R. Soc. London B, 344: 47-54.

Velázquez de Castro, A.J.; Alonso-Zarazaga, M.A. \& Outerelo, R., 1990. Curculionoidea (Coleoptera) de Navacerrada, Sierra de Guadarrama (España). Bol R. Soc. esp. Hist. Nat. (Sec. Biol.), 85(1-4): 17-37.
Viejo, J.L., Martín, J., García-Barros, E. \& Munguira, M.L., 1992. Diversidad de mariposas en el Parque Regional del Manzanares (Madrid) (Lepidoptera: Papilionoidea \& Hesperioidea). Bol. Soc. Port. Entomol., suplemento 3(1): 201-212.

WolDA, H., 1983. Diversidad de la Entomofauna y cómo Medirla. Informe Final IX Conferencia Latino-Americana de Zoología, Octubre 1983, Arequipa, Perú: 181-186.

Recibido, el 25-V-1995 Aceptado, el 18-XI-1996 Publicado, el 27-XII-1996 